\title{
LTE: Advancement of 3G Technology
}

\author{
Panta Sowmya Sri \\ M.Tech CSE \\ VNR VJIET \\ Hyderabad
}

\author{
B. V. Kiranmayee \\ Associate Professor \& HOD \\ VNR VJIET \\ Hyderabad
}

\author{
S. Nagini \\ Associate Professor \\ VNR VJIET \\ Hyderabad
}

\begin{abstract}
Long Term Evolution (LTE) is a wireless data communication technology which spread Third Generation along with the ten times pace of $3 \mathrm{G}$ networks for mobile devices and wireless communications. LTE support additional network data per user and high data rates for voice communications and high throughput. And it will support improved spectrum efficiency and service driven services. LTE is a wireless network used to provide high network performance with which streaming of media will be easy in case there is a network drop out. LTE overcomes the obstacles of second and third generation. The target of LTE is to rebuild and facilitate the network architecture to an IP based system with much latency compared to third generation architecture and its interface will be different from the $2 \mathrm{G}$ and $3 \mathrm{G}$ interfaces to facilitate high range of data communications.
\end{abstract}

\section{General Terms}

Communication Network, Wireless Mobile Network.

\section{Keywords}

LTE, Latency, Spectrum Efficiency, MIMO, OFDMA, SCFDMA

\section{INTRODUCTION}

\subsection{Second Generation (2G)}

Second Generation cellular telecom networks were economically begun on the GSM standard. $2 \mathrm{G}$ networks were based on narrow band digital networks and signals transmitted in digital format and these improve the quality of calls and reduce the complexity of data transmission. Disadvantages of $2 \mathrm{G}$ networks are weaker digital signals at less populated areas, at worst conditions the signals were no longer feasible because of these dropping of calls or the call will no longer exists and while performing digital calls, if the distance increases the voice transmission clarity reduced.

\subsection{Third Generation (3G)}

Third Generation (3G) systems for evolved to overcome the problems of $2 \mathrm{G}$ systems. After $3 \mathrm{G}$ overcrowding in existing systems will be relieved with radio spectrum, provides interoperability amid service providers and bandwidth, security, reliability is more compared to $2 \mathrm{G}$ systems. The disadvantages of $3 \mathrm{G}$ are the cost of cellular infrastructure, Roaming and data/voice can't work together, power consumption is high and requires closer base stations.

\subsection{Long Term Evolution (LTE)}

LTE is a classic for high-speed wireless communication for mobile phones and data terminals. It was started as a project in the year 2004 by a telecommunication body known as Third Generation Partnership Project (3GPP) and System Architecture Evolution (SAE) is the corresponding evolution of the general packet radio service (GPRS)/3G packet core network evolution. The term LTE is used to represent both
LTE and SAE. LTE emerged from an earlier 3GPP system which was certified as Universal Mobile Telecommunication System (UMTS), which is evolved from the Global System for Mobile Communications (GSM). Related specifications are called Evolved UMTS Terrestrial Radio Access (EUTRA) and UMTS Terrestrial Radio Access network (EUTRAN) [5]. First version of the LTE was documented in Release 8 of 3GPP specifications.

The emergence of new applications like Multimedia Online Gaming (MMOG), mobile TV etc., and a huge increase in mobile data usage have motivated the 3GPP to work on the LTE towards fourth-generation mobile to full fill the new requirements.

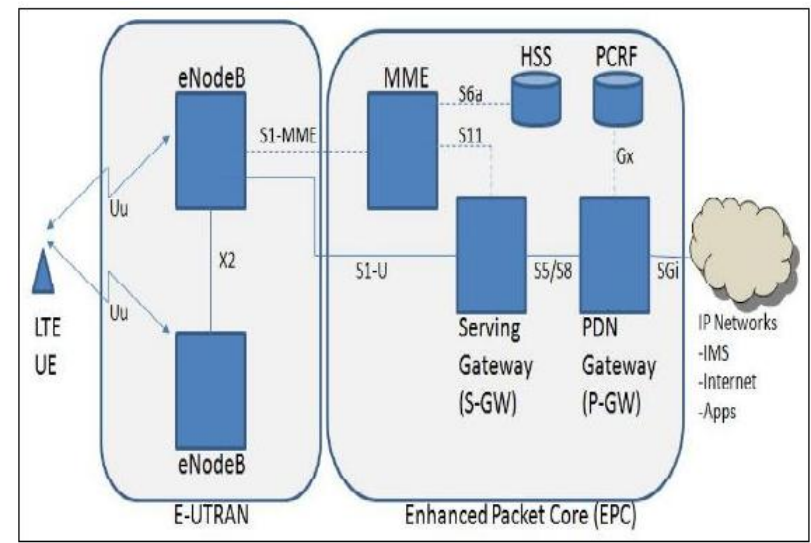

Fig 1: Architecture of LTE

The various components of LTE architecture are:

- User Equipment: The User Equipment (UE) is also called as a user's device, which has two different components, one is called as MT (Mobile Termination), which manages the communication functions like handover, media encoding and decoding etc. and TE (Terminal Equipment) which handles the termination of the data streams passes between UE and eNodeB.

- E-UTRAN: The E-UTRAN (evolved UMTS terrestrial radio access network), manages the radio communications between UE and the Enhanced Packet Core (EPC) which has one component called eNB (evolved Node B).Each eNB is known has a base station which controls the UE with in different cells. Every UE can communicate with an eNB and cells one at a time. The eNB which communicates with a UE is called as its serving eNB.

- Evolved Packet Core: The Evolved Packet Core (EPC) is a structure for providing converged voice and data over LTE network which contains Mobility Management Entity(MME) responsible for session states and authenticate to track a user with in a network, Serving 
Gateway (S-GW) routes data packets through the network, $P-G W$ (Packet Data Network Gateway) is the interface between LTE network and other packet data networks (PDNs) and regulates QoS (quality of service)and PCRF (Policy and Charging Rules Function) supports the policy enforcement to meet the requirements of the network, service data flow detection which detects which type of services the user is requested or accessed and service charges will be maintained based on the data flow.

\section{LTE PROTOCOLS}

LTE Protocol stack is associated with interfaces which the elements of network use to exchange data and signaling of messages. The basic architecture of LTE protocol is as shown below:

\begin{tabular}{|c|c|}
\hline \multicolumn{1}{|c|}{$\begin{array}{c}\text { User plane } \\
\text { (Data) }\end{array}$} & $\begin{array}{c}\text { Control plane } \\
\text { (LTE signalling) }\end{array}$ \\
\hline $\begin{array}{c}\text { User plane } \\
\text { protocols }\end{array}$ & $\begin{array}{c}\text { Signalling } \\
\text { protocols }\end{array}$ \\
\hline Transport protocols \\
\hline
\end{tabular}

Fig 2: Architecture of LTE Protocols

The protocol has two components User Plane and Control Plane. User Plane protocols handles data that are passed by the user and Control Plane protocols handles the signaling messages that are used by the network elements.

\subsection{User Plane Protocol Stack}

The User Plane Protocol Stack will be in between UE and eNodeB. Where it contains sub layers Packet Data Convergence Protocol (PDCP) handles high level transport functions like header compression and security, Radio Link Control (RLC) provides a data link between devices and Medium Access Control (MAC) which handles the low level control of physical layer like data transmissions between UE and eNodeB. The protocol stack for LTE User plane will be as shown below:

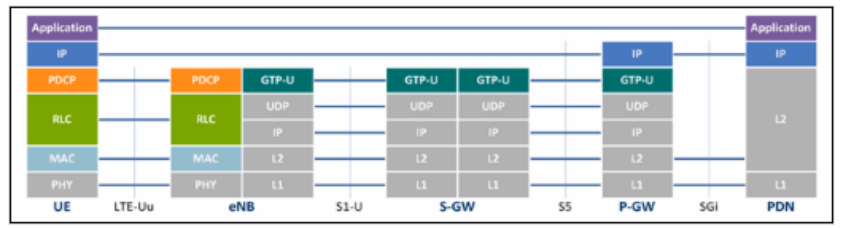

Fig 3: User Plane Control Stack

The interfaces provided by the user plane are LTE-Uu, S1-U, S5 and SGI.

- $\quad$ LTE-Uu interface: Through this interface PDCP, RLC and MAC will transfer data from UE to eNodeB.

- S1/S5/SGI Interface: Through this interfaces GTP-U (GPRS Tunneling Protocol User part) protocol forward user IP Packets.

\subsection{Control Plane Protocol Stack}

The following Figure 4 represents the Control Plane Protocol Stack:

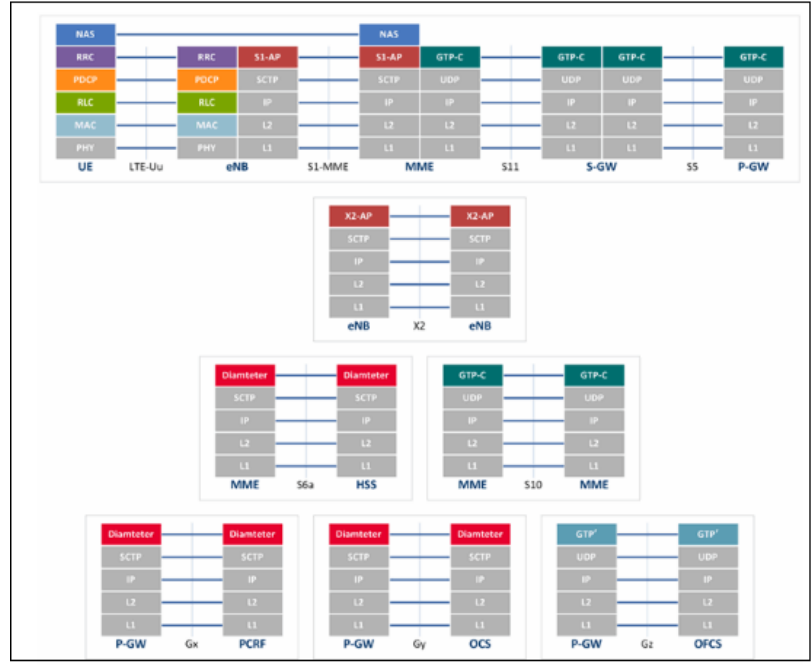

Fig 4: Control Plane Protocol Stack

The following are the interfaces provided by the control plane:

- LTE-Uu Interface: The NAS (Non Access Stratum) protocol handles mobility and bearer management functions. RRC (Radio Resource Control) protocol supports the transfer of signals provided by NAS, performs broadcasting, setup, modifies and release of radio bearer and also reconfigures, reestablish the RRC connection.

- X2 Interface: Through X2 interface X2AP protocols support UE movability and SON (Self Optimizing Network) functions within E-UTRAN. User data forwarding, SN status transfer and UE context release are the functions provided by X2ap protocol for UE movability. As SON functions, eNB interchange resource status, traffic load, configuration update information and correlative with each other for mobility parameters.

- S1-MME Interface: Through S1-MME interface S1ap protocol supports functions like $\mathrm{S} 1$ interface management, E-RAB management, NAS signaling transport and UE context management.

- S11/S5/S10 Interfaces: Through these interfaces GTP-C (GPRS Tunneling Protocol Control part) protocol handles interchange of control information for creation, modification and termination of GTP Tunnels.

- S6a Interface: Through this interface Diameter protocol handles interchange of the subscriber and subscription authentication information between HSS and MME.

- Gx Interface: The Diameter protocol handles delivery of PCC rules from PCRF to P-GW over this interface.

- Gy Interface: Through this interface diameter protocol supports interchange of the real-time control information between OCS and P-GW.

Gz Interface: The GTP protocol handles CDR transfer from $\mathrm{P}-\mathrm{GW}$ to the OFCS over this interface [4].

\section{CHANNELS AND SIGNALS}

The information flows between different LTE protocols are called channels and signals. Different types of channels are logical and transport channels. Logical channels carries data and signaling messages between RLC and MAC protocols. 
Transport channels carries data and signaling messages between MAC and Physical layer.

The most crucial logical channels are Dedicated Traffic Channel (DTCH) carries data to or from the device, Dedicated Control Channel (DCCH) carries signaling messages, and Broadcast Control Channel (BCCH) carries RRC messages information, these are divided into two parts. One is Master Information Block (MIB) and another is SIB which is of 11types. They are described below:

- MIB: It transmits Bandwidth, PHICH information, system frame number and spare.

- SIB-1: It Contains information about LTE cell and Carries cell access related parameters and defines scheduling of other SIBs

- SIB-2: It Carries Common, shared and RACH channel configuration

- SIB-3: It Carries cell and Intra frequency cell reselection information

- SIB-4: It Carries Information about INTRA-frequency neighbouring cells

- SIB-5: It Carries Information about INTER-frequency neighbouring cells

- SIB-6: It Carries WCDMA information that carries UTRA and neighbour cell frequencies which are useful for cell re-selection.

- SIB-7: Carries GSM neighbours information such as GERAN frequencies and GERAN neighbour cell frequencies and is used for cell reselection

- SIB-8: It Carries CDMA-2000 EVDO, CDMA-2000 neighbour cell frequencies

- SIB-9: It Carries Information about Home eNodeB

- SIB-10: It Carries Earthquake and Tsunami Warning System (ETWS) information as Primary Notification

- SIB-11: It Carries Earthquake and Tsunami Warning System (ETWS) information as a notification [5].

\section{NEED FOR LTE}

- Using LTE a user can open a browser and download high bandwidth media quickly.

- As LTE downloads the media faster, connection with the network gets released faster for each connection. This will reduces the traffic load in the network.

- $\quad$ LTE has an improved architecture due to this handoff is smooth from one region to another region, this results into continuous data streaming without interruption on on-going data transfer.

\section{CONCLUSIONS}

In this paper, LTE communication network is introduced which overcome the obstacles of $3 \mathrm{~g}$ and $2 \mathrm{G}$ networks. LTE provides MIMO with this high data rates achieved, it uses OFDMA in the downlink so that capacity of the network increases because of these different users can utilize different channels to access the system and uses SC-FDMA in uplink hence mobile terminals can have low power during data transmission which increases the battery life at users end.

\section{REFERENCES}

[1] S. Tomazic and G. Jakus, "Long term evolution: Towards 4th generation of mobile telephony and beyond", 9th Int. Conf. on Telecommunication in Modern Satellite Cable and Broadcasting Services, pp. 91-96, 2009.

[2] H. Ghazzai, E. Yaacoub, M.-S. Alouini and A. AbuDayya, "Optimized green operation of lte networks in the presence of multiple electricity providers", Proc. IEEE International Workshop on Emerging Technologies for LTE-Advanced and Beyond-4G in Conjunction with IEEE Global Communications Conference (Globecom 2012), 2012.

[3] 3GPP TS 36.211 3GPP TSG RAN Evolved Universal Terrestrial Radio Access (E-UTRA) Physical Channels and Modulation Version 11.0.0 Release 11, 2012.

[4] Yi, SeungJune, SungDuck Chun, YoungDae Lee, SungJun Park, and SungHoon Jung. Radio Protocols for LTE and LTE-advanced. John Wiley \& Sons, 2012.

[5] Cox, Christopher. An introduction to LTE: LTE, LTEadvanced, SAE and 4G mobile communications. John Wiley \& Sons, 2012. 